\title{
Boning up on blood
}

The contribution of the microenvironment to oncogenesis has been extensively reported. Raaijmakers et al. have made a valuable contribution to this field by describing how specific changes in the microenvironment can initiate cancer development by triggering secondary genetic changes in other cells.

Mesenchymal stromal cells are a constituent of almost all tissues and take part in organ formation and homeostasis. Working on the principle that mesenchymal cells of the osteoblast lineage modulate haematopoiesis, the authors investigated which stage of differentiation of osteoblastic cells was crucial for the regulation of haematopoiesis. To that end, they deleted Dicer 1 , a gene required for RNA and microRNA processing, so that many of the resulting gene products would be altered. Mice lacking Dicer1 in osteoprogenitors, but not mature osteoblasts, showed severely altered haematopoiesis, including cytopenia, multilineage dysplasia, increased proliferation and apoptosis of primitive haematopoietic progenitors, decreased B cell levels and increased bone marrow vascularity, all of which are principle characteristics of myelodysplastic syndrome. In addition, a small proportion of the animals developed facial myeloid sarcomas and acute myelogenous leukaemia.

To assess how much the microenvironment contributes to this haematopoietic phenotype, the authors then transplanted bone marrow cells of Dicer1mutant mice into wild-type mice. Analysis of the bone marrow and peripheral blood 16 weeks after the transplant showed complete normalization of haematopoiesis. By contrast, when bone marrow cells from wild-type mice were transplanted into mice with the Dicer1-deleted microenvironment, the recipients developed haematopoietic abnormalities.

Microarray analysis of primary osteolineage cells lacking Dicer1 showed decreased expression of $\underline{S b d s}$, a gene mutated in Schwachman-Bodian-Diamond syndrome, a condition that is characterized by haematopoietic abnormalities as well as a propensity to develop myelodysplastic syndrome and acute myelogenous leukaemia. Accordingly, when the authors deleted Sbds in osteoprogenitor cells they obtained a similar phenotype to that observed when

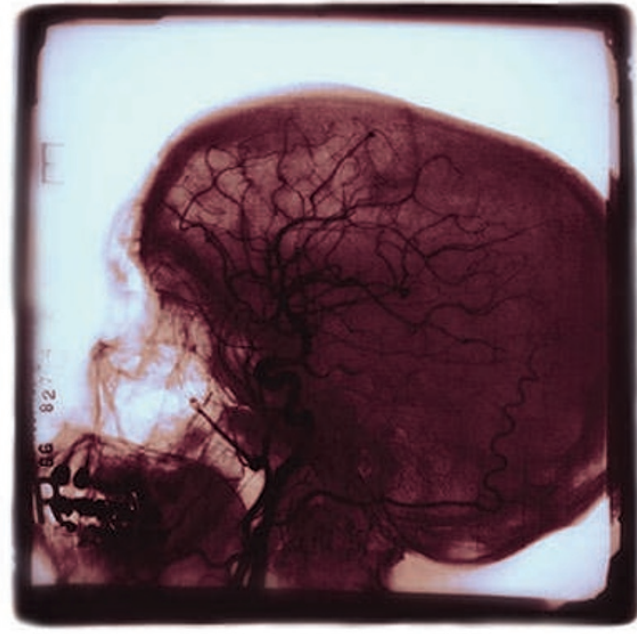

deleting Dicer1, with resulting severe bone marrow dysfunction and myelodysplasia.

These findings demonstrate that alterations in a specific mesenchymal microenvironment can be sufficient to initiate a disordered haemostasia that can result in cancer initiation. Raaijmakers et al. postulate a niche-based model of oncogenesis in which the microenvironment becomes the starting point for a series of genetic changes that lead to malignancy in a different type of cell.

Teresa Villanueva

ORIGINAL RESEARCH PAPER Raaijmakers, M. H. G. P. et al. Bone progenitor dysfunction induces myelodysplasia and secondary leukaemia. Nature 21 Mar 2010 (doi:10.1038/nature08851) 